\title{
Effect of temozolomide on survival in elderly patients with glioblastoma and impaired
} performance status: a propensity score-matching analysis

\author{
This article was published in the following Dove Press journal: \\ OncoTargets and Therapy \\ 14 August 2017 \\ Number of times this article has been viewed
}

\section{Yong-Liang Liu \\ Peng-Fei Liu \\ Wei Shao \\ Hong-Peng Du \\ Zhen-Zhu Li \\ Chong Guo \\ Ze-Fu Li}

Department of Neurosurgery, Affiliated Hospital of Binzhou Medical University, Binzhou, Shandong, Yantai,

People's Republic of China
Correspondence: Ze-Fu Li Department of Neurosurgery, Affiliated Hospital of Binzhou Medical University, Binzhou, Shandong, Yantai 256600,

People's Republic of China

Tel/fax +865433258538

Email lizefubz@163.com
Objective: At present, there is no consensus regarding the standard treatment for glioblastoma (GBM) in elderly patients with impaired Karnofsky performance status (KPS) scores. This study aimed to determine the effects of temozolomide (TMZ) versus best supportive care (BSC) in this population.

Materials and methods: We conducted a retrospective observational study of patients aged $\geq 65$ years with histologically confirmed GBM and KPS scores $\leq 70$ who were treated at our institution between January 2006 and July 2014. Demographic data, treatments, and outcomes were evaluated. Univariate and multivariate analyses were performed to identify the independent prognostic factors of overall survival (OS) and progression-free survival (PFS). The impact of TMZ on survival was analyzed by the application of propensity score matching of clinicopathological factors among patients who received TMZ vs BSC.

Results: There were 153 patients ( 86 men, 56.2\%) in this study. The median patient age was 70 years (range: $65-83$ years). The median KPS score was 60 (range: 30-70). Seventy-eight patients $(51.0 \%)$ received TMZ, whereas 75 (49.0\%) received BSC. Median OS and PFS were 6.0 and 4.5 months, respectively. Compared with BSC, TMZ was associated with improved OS (hazard ratio [HR]: $0.38,95 \% \mathrm{CI}: 0.17-0.70 ; P=0.002$ ) and PFS (HR: $0.41,95 \% \mathrm{CI}: 0.21-0.76$; $P=0.003)$ after propensity score matching. Factors independently associated with OS were KPS score (HR: 2.11,95\% CI: 1.48-7.67; $P=0.016$ ), extent of resection (HR: 1.98, 95\% CI: 1.45-5.14; $P=0.026$ ), and treatment group (HR: $0.49,95 \% \mathrm{CI}: 0.23-0.87 ; P=0.019$ ). The most frequent toxicity in the TMZ group was myelosuppression.

Conclusion: Compared with BSC, TMZ increased survival with acceptable toxicity in elderly GBM patients with KPS scores $\leq 70$.

Keywords: temozolomide, best supportive care, elderly, glioblastoma, prognostic factors

\section{Introduction}

Glioblastoma (GBM) is the most frequent and most aggressive primary brain malignancy, with an incidence of $>3$ per 100,000 individuals per year. ${ }^{1}$ In addition, the incidence rate of GBM in patients aged $>65$ years is increasing rapidly, with reported incidence rates of up to 10.6 per 100,000 individuals in this age cohort. ${ }^{2}$ The number of elderly patients with GBM is expected to increase in the near future as the elderly population increases. Several studies have revealed a survival benefit of adjuvant radiotherapy and chemotherapy in elderly GBM patients with a good performance status. ${ }^{3-5}$ However, the optimal management strategy for elderly GBM patients with 
impaired performance status (Karnofsky performance status [KPS] score $\leq 70$ ) has not yet been determined because of the paucity of data available from this fragile population. Furthermore, owing to the intolerance to radiotherapy and the short expected survival times, these patients frequently receive only best supportive care (BSC). ${ }^{6}$

Temozolomide (TMZ) is an alkylating imidazole tetrazine derivative that is well tolerated in GBM patients. A recent study has revealed that $\mathrm{TMZ}$ prolongs survival and hence should be considered as a standard treatment option in elderly patients with GBM. ${ }^{7}$ We undertook the present study to determine the effectiveness of TMZ relative to BSC in improving the prognosis of elderly patients with GBM and KPS scores of $\leq 70$. In addition, we conducted a propensity score-matching analysis to adjust for between-group disparities of clinically relevant covariates.

\section{Materials and methods}

\section{Patient cohort}

We retrospectively reviewed the medical records of 153 patients aged $\geq 65$ years who were histologically confirmed to have GBM in the Affiliated Hospital of Binzhou Medical University between January 2006 and July 2014. The inclusion criteria included the following: 1) histologically proven GBM according to the WHO classification; 2 ) age $\geq 65$ years; 3 ) KPS score $\leq 70$; and 4 ) no history of other malignancies. The medical records of each patient were reviewed, and various patient, tumor, and treatment variables were noted. Patient demographic characteristics, comorbidities, KPS scores, tumor characteristics, and therapies delivered during the first course of treatment were recorded. The Charlson weighted index of comorbidities (WIC) was applied to obtain a descriptive analysis of this cohort's comorbidity burden. ${ }^{8}$ Tissue samples and clinical data were available for all patients. The DNA methylation status of the $M G M T$ promoter was determined using a methylationspecific polymerase chain reaction method. ${ }^{9}$ All participants gave written informed consent to participate in this study, and this study was approved by the ethics committee of the Affiliated Hospital of Binzhou Medical University in the People's Republic of China.

All patients underwent surgery followed by adjuvant chemotherapy or BSC. The surgeries were categorized as radical resection, partial resection, or biopsy only. The first cycle of adjuvant TMZ was administered at a dose of $150 \mathrm{mg} / \mathrm{m}^{2}$ (days 1-5) and, depending on the tolerance, this was increased to $200 \mathrm{mg} / \mathrm{m}^{2}$ in the next cycle for a minimum of six cycles every 4 weeks.
After completion of the treatment, the patients were regularly followed up. Acute toxicity was evaluated according to the Common Terminology Criteria for Adverse Events version 3.0 (National Cancer Institute; National Institutes of Health, Bethesda, MD, USA). The date of last follow-up was May 31, 2016.

\section{Statistical analysis}

Differences in baseline characteristics were assessed using the $\chi^{2}$ test in the case of categorical variables and the twosided $t$-test in the case of continuous variables. The logistic regression model was applied to assess associations between clinicopathological factors and TMZ treatment and to derive propensity scores. Overall survival (OS) was defined as the duration from the diagnosis of GBM until death or last follow-up. Progression-free survival (PFS) was defined as the duration from the diagnosis of GBM until the last follow-up, the detection of locoregional recurrence or distant progression, or death. Univariate analyses were carried out using Kaplan-Meier plots and log-rank statistics. Variables at significance levels of $P<0.1$ on univariate analysis were included in a multivariate analysis. Multivariate analyses using the Cox proportional hazards model were applied to identify the independent prognostic factors. To eliminate bias introduced by the nonrandomized treatment assignment, patients (TMZ group) and controls (BSC group) were matched $1: 1$ by the nearest-neighbor method, using a caliper distance of $15 \%$ of the SD of the estimated propensity score logit to ensure good matches. ${ }^{10}$ The following independent variables were included in the model, regardless of individual statistical significance: age at diagnosis, KPS score, sex, time of diagnosis, WIC, MGMT methylation status, serum albumin level, and extent of resection. A competing-risks analysis was applied to compare the different types of deaths between the TMZ and BSC groups. The Gray test was applied to compare the cumulative incidence functions estimated in the competing risk analysis. ${ }^{11}$ Statistical analyses were performed using the SPSS 18.0 software (IBM, Armonk, NY, USA) and R version 3.2.2 (Institute for Statistics and Mathematics, Vienna, Austria; www.R-project.org) with R packages rms and cmprsk. $P$-values $<0.05$ were considered statistically significant.

\section{Results}

\section{Patient characteristics}

A total of 153 consecutive patients aged $\geq 65$ years with histologically confirmed GBM and a postoperative KPS score of $\leq 70$ were reviewed in this study. The patient 
characteristics are summarized in Table 1 . Of the 153 patients, $86(56.2 \%)$ were men and $67(43.8 \%)$ were women. In total, 78 patients $(51.0 \%)$ received TMZ and $75(49.0 \%)$ received BSC. The median age was 69 years (range: 65-74 years) in the TMZ group and 73 years (range: 65-83 years) in the BSC group. The median KPS score in the entire cohort was 60 (range: $30-70$ ). The median symptom duration was 6 months (range: $1-10$ months), and the most frequent symptoms were those caused by increased intracranial pressure (eg, headache and vomiting), which occurred in 91 patients $(59.5 \%)$. Tumors were most frequently located in the frontal lobe (53 patients, 34.6\%). Forty-eight (31.4\%) patients had temporal lobe invasion. Nine patients $(5.9 \%)$ had multilobar involvement ( $\geq 2$ lobes involved). All patients underwent surgery; macroscopic radical resection was performed in 64 patients $(41.8 \%)$; partial resection was performed in eight patients $(5.2 \%)$; and biopsy only was conducted in 81 patients $(53.0 \%)$. There were no major comorbidities or postoperative complications in any of the patients.

Patients in the TMZ group received a median of three cycles of TMZ (range: 1-12 cycles). Dose delays occurred in 19 patients $(24.3 \%)$, and dose reductions were required in 21 patients $(26.9 \%)$ due to treatment-related toxicities, such as myelosuppression. There were no statistically significant differences between the two groups in terms of sex $(P=0.517)$, KPS score $(P=0.716), M G M T$ methylation status $(P=0.746)$, serum albumin level $(P=0.901)$, and extent of resection $(P=0.416)$. The difference in the WIC was close to - but did not reach - statistical significance $(P=0.076)$. Patients in the TMZ group were significantly more likely to be younger $(P=0.015)$ and be diagnosed between 2012 and 2014 ( $P=0.013$; Table 1$)$.

\section{Survival outcomes and propensity score- matching analysis}

The median OS duration was 6.0 months, and the median PFS duration was 4.5 months in the entire cohort. After propensity score matching, 122 patients were available for outcome comparison. In the propensity score-matched cohort, the TMZ group had significantly better OS than the BSC group ( $15.4 \%$ vs $9.8 \%$ after 1 year, $P=0.002$; Figure $1 \mathrm{~A}$ ). Similarly, PFS was significantly better in the TMZ group than in the

Table I Baseline patient and treatment characteristics in the observational data set and in patients after propensity score matching

\begin{tabular}{|c|c|c|c|c|c|c|}
\hline \multirow[t]{3}{*}{ Characteristics } & \multicolumn{3}{|c|}{ Observational data set $(n=\mid 53)$} & \multicolumn{3}{|c|}{ Propensity score-matched data set $(n=\mid 22)$} \\
\hline & \multirow{2}{*}{$\frac{T M Z}{n=78(\%)}$} & \multirow{2}{*}{$\frac{\text { BSC }}{n=75(\%)}$} & \multirow[t]{2}{*}{$P$-value } & \multirow{2}{*}{$\frac{T M Z}{n=6 I(\%)}$} & \multirow{2}{*}{$\frac{\text { BSC }}{n=6 \text { I (\%) }}$} & \multirow[t]{2}{*}{$P$-value } \\
\hline & & & & & & \\
\hline Age at diagnosis, years & - & - & 0.015 & - & - & 0.587 \\
\hline $65-70$ & $47(60.3)$ & $30(40.0)$ & - & $33(54.1)$ & $29(47.5)$ & - \\
\hline$>70$ & $31(39.7)$ & $45(60.0)$ & - & $28(45.9)$ & $32(52.5)$ & - \\
\hline KPS score & - & - & 0.706 & - & - & 0.716 \\
\hline 60-70 & $45(57.7)$ & $4 \mathrm{I}(54.7)$ & - & $34(55.7)$ & $32(52.5)$ & - \\
\hline$<60$ & $33(42.3)$ & $34(45.3)$ & - & $27(44.3)$ & $29(47.5)$ & - \\
\hline Sex & - & - & 0.517 & - & - & 0.856 \\
\hline Male & $46(58.9)$ & $40(53.3)$ & - & $32(52.5)$ & $31(50.8)$ & - \\
\hline Female & $32(4 I . I)$ & $35(46.7)$ & - & $29(47.5)$ & $30(49.2)$ & - \\
\hline Time of diagnosis & - & - & 0.013 & - & - & 0.760 \\
\hline 2006-2008 & $19(24.4)$ & $3 I(4 I .3)$ & - & 17 (27.9) & $19(31.1)$ & - \\
\hline $2009-2011$ & $21(26.9)$ & $24(32.0)$ & - & $18(29.5)$ & $20(32.8)$ & - \\
\hline $2012-2014$ & $38(48.7)$ & $20(26.7)$ & - & $26(42.6)$ & $22(36.1)$ & - \\
\hline WIC & - & - & 0.076 & - & - & 0.365 \\
\hline$\geq 3$ & $31(39.7)$ & $4 \mid(54.7)$ & - & $27(44.3)$ & $33(54.1)$ & - \\
\hline$<3$ & $47(60.3)$ & $34(45.3)$ & - & $34(55.7)$ & 28 (45.9) & - \\
\hline MGMT methylation status & - & - & 0.746 & - & - & 0.856 \\
\hline Methylated & $33(42.3)$ & $34(45.3)$ & - & $29(47.5)$ & $30(49.2)$ & - \\
\hline Unmethylated & $45(57.7)$ & $4 \mid(54.7)$ & - & $32(52.5)$ & $31(50.8)$ & - \\
\hline Serum albumin, g/L & - & - & 0.901 & - & - & 0.810 \\
\hline$<35$ & $12(15.4)$ & II (I4.7) & - & II (I8.0) & $10(16.4)$ & - \\
\hline$\geq 35$ & $66(84.6)$ & $64(85.3)$ & - & $50(82.0)$ & $51(83.6)$ & - \\
\hline Extent of resection & - & - & 0.416 & - & - & 0.717 \\
\hline Radical resection & $30(38.5)$ & $34(43.6)$ & - & $27(44.3)$ & $30(49.2)$ & - \\
\hline Partial resection/biopsy & $48(6 \mid .5)$ & $4 \mid(54.7)$ & - & $34(55.7)$ & $31(50.8)$ & - \\
\hline
\end{tabular}

Abbreviations: BSC, best supportive care; KPS, Karnofsky performance status; TMZ, temozolomide; WIC, Charlson weighted index of comorbidities. 

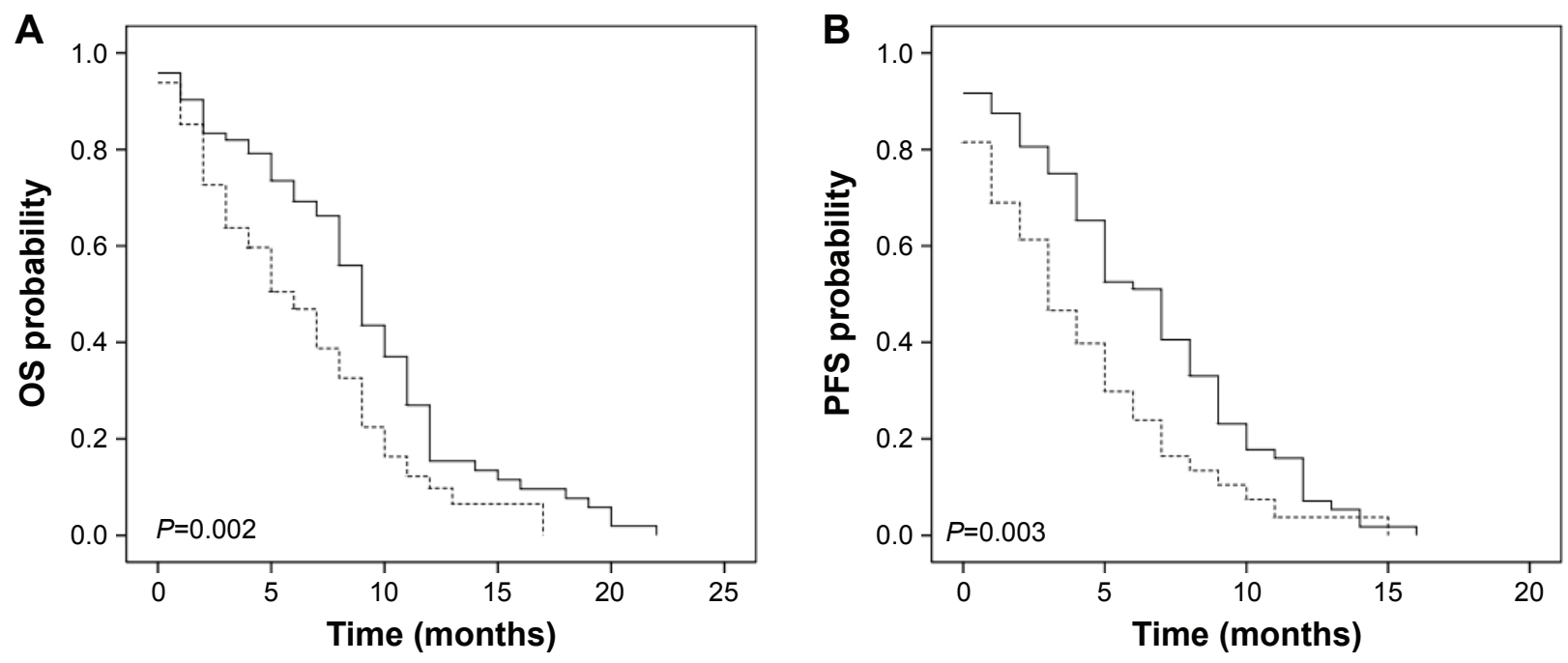

\section{$\neg$ TMZ}

Figure I Kaplan-Meier estimated curves of (A) OS and (B) PFS in patients receiving TMZ vs BSC.

Abbreviations: BSC, best supportive care; OS, overall survival; PFS, progression-free survival; TMZ, temozolomide.

BSC group ( $9.8 \%$ vs $3.7 \%$ after 1 year, $P=0.003$; Figure $1 \mathrm{~B}$ ). Univariate survival analysis in the propensity score-matched cohort showed that the following factors were significantly associated with worse OS (Table 2): age $>70$ years (hazard ratio [HR]: $1.39,95 \% \mathrm{CI}: 1.02-2.28 ; P=0.031)$, KPS score $<60$ (HR: 2.73, 95\% CI: 1.58-7.29; $P=0.003$ ), partial resection/biopsy (HR: 2.69, 95\% CI: 1.59-6.39; $P=0.003$ ), and BSC (HR: $0.38,95 \%$ CI: $0.17-0.70 ; P=0.002$ ). The following variables were associated with significantly worse PFS on univariate analysis: KPS score $<60$ (HR: 3.11, 95\% CI: $1.89-11.34 ; P=0.001)$, unmethylated $M G M T$ promoter (HR: $0.29,95 \%$ CI: $0.13-0.88 ; P=0.006)$, partial resection/ biopsy (HR: 4.15, 95\% CI: 2.34-10.46; $P<0.001$ ), and BSC (HR: $0.41,95 \%$ CI: $0.21-0.76 ; P=0.003$ ). The statistically significant factors in the univariate analysis $(P<0.10)$ were further analyzed using multivariate survival analysis.

In the multivariate Cox proportional hazards regression model, KPS score (HR: 2.11, 95\% CI: 1.48-7.67; $P=0.016$ ), extent of resection (HR: $1.98,95 \% \mathrm{CI}: 1.45-5.14 ; P=0.026$ ), and treatment group (HR: $0.49,95 \%$ CI: $0.23-0.87$; $P=0.019)$ were identified as significant prognostic factors for OS (Table 2). KPS score (HR: 2.15, 95\% CI: 1.18-8.33; $P=0.004$ ), $M G M T$ methylation status (HR: $0.33,95 \% \mathrm{CI}$ : $0.26-0.89 ; P=0.013$ ), extent of resection (HR: $3.76,95 \%$ CI: $2.58-7.36 ; P=0.002$ ), and treatment group (HR: 0.56 , 95\% CI: $0.29-0.81 ; P=0.025)$ were significant prognostic factors for PFS.

In addition, the cumulative incidence functions for cancer events significantly differed between the two groups
(Gray test, $P=0.025$ ), but the cumulative incidence of the competing event (documented noncancer death) was not significantly different (Gray test, $P=0.263$ ) in the competing risk analysis.

\section{Toxicity}

The TMZ cohort ( $\mathrm{n}=78$ ) was evaluated for toxicity. Most documented acute toxicities were either mild or moderate. The acute hematological toxicities that occurred in the TMZ group are listed in Table 3. Nonhematological adverse events of grade $\geq 3$ consisted of cutaneous reactions (four patients, $5.1 \%$ ) and hepatic toxicity (three patients, 3.8\%). Thromboembolic events occurred in two patients $(2.7 \%)$.

\section{Discussion}

The incidence of GBM has dramatically increased in the elderly population. ${ }^{12}$ To date, the standard treatment for elderly GBM patients remains undefined and controversial. For elderly GBM patients with poor KPS scores, reasonable treatment options include BSC and TMZ monotherapy. ${ }^{13}$ The findings of the present study revealed that TMZ monotherapy showed a survival advantage over BSC in elderly GBM patients with poor KPS scores. This finding was supported by the results of the propensity score-matching analysis, which can address potential bias when retrospective data of two nonrandomized groups are compared.

The propensity score enables the estimation of the probability of undergoing a treatment given a vector of observed factors. Compared with conventional case-control 
Table 2 Univariate and multivariate analyses in the propensity score-matched cohort $(n=122)$

\begin{tabular}{|c|c|c|c|c|c|c|c|c|}
\hline \multirow[t]{3}{*}{ Factors } & \multicolumn{4}{|l|}{ OS } & \multicolumn{4}{|l|}{ PFS } \\
\hline & \multicolumn{2}{|c|}{ Univariate analysis } & \multicolumn{2}{|c|}{ Multivariate analysis } & \multicolumn{2}{|c|}{ Univariate analysis } & \multicolumn{2}{|c|}{ Multivariate analysis } \\
\hline & HR (95\% Cl) & $P$-value & HR $(95 \% \mathrm{Cl})$ & $P$-value & HR (95\% Cl) & $P$-value & HR (95\% Cl) & $P$-value \\
\hline \multicolumn{9}{|l|}{ Age, years } \\
\hline 65-70 & Ref & - & Ref & - & Ref & - & - & - \\
\hline$>70$ & $1.39(1.02-2.28)$ & 0.031 & $1.26(0.89-1.69)$ & 0.148 & $1.19(0.68-2.85)$ & 0.170 & - & - \\
\hline \multicolumn{9}{|l|}{ KPS score } \\
\hline 60-70 & Ref & - & Ref & - & Ref & - & Ref & - \\
\hline$<60$ & $2.73(1.58-7.29)$ & 0.003 & $2.11(1.48-7.67)$ & 0.016 & $3.11(1.89-11.34)$ & 0.001 & $2.15(1.18-8.33)$ & 0.004 \\
\hline \multicolumn{9}{|l|}{ Sex } \\
\hline Male & Ref & - & - & - & Ref & - & - & - \\
\hline Female & $0.81(0.53-1.19)$ & 0.259 & - & - & $0.75(0.26-1.33)$ & 0.332 & - & - \\
\hline \multicolumn{9}{|l|}{ Time of diagnosis } \\
\hline 2006-2008 & Ref & - & - & - & Ref & - & - & - \\
\hline $2009-2011$ & I.28 (0.78-I.68) & 0.268 & - & - & $1.13(0.75-1.54)$ & 0.549 & - & - \\
\hline $2012-2014$ & $0.86(0.56-1.36)$ & 0.093 & - & - & $0.89(0.63-1.69)$ & 0.186 & - & - \\
\hline \multicolumn{9}{|l|}{ WIC } \\
\hline$\geq 3$ & Ref & - & - & - & Ref & - & - & - \\
\hline$<3$ & $0.69(0.59-1.15)$ & 0.158 & - & - & $0.81(0.52-1.33)$ & 0.258 & - & - \\
\hline \multicolumn{9}{|l|}{ MGMT methylation status } \\
\hline Methylated & Ref & - & - & - & Ref & - & Ref & - \\
\hline Unmethylated & $0.65(0.36-1.36)$ & 0.169 & - & - & $0.29(0.13-0.88)$ & 0.006 & $0.33(0.26-0.89)$ & 0.013 \\
\hline \multicolumn{9}{|l|}{ Serum albumin, g/L } \\
\hline$<35$ & Ref & - & - & - & Ref & - & - & - \\
\hline$\geq 35$ & $0.89(0.62-1.16)$ & 0.158 & - & - & $0.93(0.78-1.32)$ & 0.328 & - & - \\
\hline \multicolumn{9}{|l|}{ Extent of resection } \\
\hline Radical resection & Ref & - & Ref & - & Ref & - & Ref & - \\
\hline Partial resection/biopsy & $2.69(1.59-6.39)$ & 0.003 & $1.98(1.45-5.14)$ & 0.026 & $4.15(2.34-10.46)$ & $<0.001$ & $3.76(2.58-7.36)$ & 0.002 \\
\hline \multicolumn{9}{|l|}{ Treatment } \\
\hline TMZ & $0.38(0.17-0.70)$ & 0.002 & $0.49(0.23-0.87)$ & 0.019 & $0.4 I(0.21-0.76)$ & 0.003 & $0.56(0.29-0.8 \mathrm{I})$ & 0.025 \\
\hline BSC & Ref & - & Ref & - & Ref & - & Ref & - \\
\hline
\end{tabular}

Abbreviations: BSC, best supportive care; HR, hazard ratio; Ref, reference; KPS, Karnofsky performance status; OS, overall survival; PFS, progression-free survival; TMZ, temozolomide; WIC, Charlson weighted index of comorbidities.

adjustments, the propensity score-matching analysis is a powerful tool that can be used to draw causal inferences from observational data. ${ }^{14}$ By comparing the TMZ and BSC groups by using propensity score-matching analysis, we were able to control for important prognostic factors, all of which were associated with GBM characteristics and outcomes. In addition, we restricted the analysis to patients aged $\geq 65$ years with KPS scores $\leq 70$ because age and KPS score have been reported to play prognostic roles in GBM. ${ }^{15-17}$ Patients aged $>60$ years at the time of diagnosis

Table 3 Acute hematological toxicity (TMZ group, $n=78$ )

\begin{tabular}{llllll}
\hline Toxicities & \multicolumn{5}{l}{ CTC grade } \\
\cline { 2 - 6 } & $\mathbf{0}$ & $\mathbf{I}$ & $\mathbf{2}$ & $\mathbf{3}$ & $\mathbf{4}$ \\
\hline Anemia & 32 & 28 & 15 & 3 & 0 \\
Leukocytopenia & 30 & 23 & 16 & 6 & 3 \\
Neutropenia & 14 & 34 & 25 & 3 & 2 \\
Thrombocytopenia & 15 & 26 & 29 & 8 & 2 \\
\hline
\end{tabular}

Abbreviations: CTC, Common Terminology Criteria; TMZ, temozolomide. have significantly worse prognosis than GBM patients aged $<45$ years. ${ }^{15}$ In addition, GBM patients with KPS scores $\geq 90$ have significantly longer survival times than patients with KPS scores $<90 .{ }^{17}$ Using these measures, we were able to minimize both the potential bias introduced by these important confounders and the inherent selection bias of a retrospective study.

Currently, the standard therapy for elderly GBM patients with poor KPS scores is not well established. Because radiotherapy requires daily trips to the hospital and induces fatigue, it is considered inconvenient for these severely ill patients with short survival expectancy. In addition, tolerance to radiotherapy remains an important concern in these patients. Considering the lack of studies addressing the role of chemotherapy in this population, elderly patients are less likely to receive multiagent treatment and frequently receive only BSC. ${ }^{6} \mathrm{TMZ}$ is an alkylating imidazole tetrazine derivative, which inhibits DNA replication and triggers cancer cell death. Several recent studies have revealed that TMZ alone 
could be a reasonable alternative to radiotherapy in elderly GBM patients. ${ }^{7,18}$ A meta-analysis comparing TMZ alone vs radiotherapy in elderly patients with GBM found that OS was significantly longer in the TMZ group than in the radiotherapy group, ${ }^{19}$ indicating that $\mathrm{TMZ}$ alone is an effective treatment option, especially in patients with $M G M T$ promoter methylation. ${ }^{19,20}$ Perez-Larraya et $\mathrm{al}^{6}$ conducted a nonrandomized Phase II study, which enrolled 70 GBM patients aged at least 70 years with KPS scores $<70$. The study revealed that compared with BSC, TMZ could improve the functional status and appeared to increase survival. ${ }^{6}$ Another nonrandomized Phase II trial assessed the efficacy and safety of TMZ plus bevacizumab in elderly GBM patients with poor performance status and reported that the median OS time was 24 weeks, with tolerable toxicity. ${ }^{21}$ Our study confirmed the survival advantage of $\mathrm{TMZ}$ relative to BSC only in elderly GBM patients with poor KPS scores. The TMZ group had a significantly better OS than the BSC group, and this benefit of TMZ remained significant in the multivariate models and after propensity score-matching adjustments for important clinical variables, notably age at diagnosis, KPS score, sex, time of diagnosis, MGMT methylation status, WIC score, serum albumin level, and extent of resection. The median OS duration was 6.0 months, and the median PFS duration was 4.5 months in the entire cohort, which is consistent with previous studies. ${ }^{6,19}$

The $M G M T$ gene encodes a DNA repair enzyme that could abrogate the alkylating effect of $\mathrm{TMZ}$ and reduce its clinical efficacy. ${ }^{22}$ Approximately $40 \%-45 \%$ of GBM tumors exhibit hypermethylation of the $M G M T$ promoter region, which inactivates the $M G M T$ gene and increases the clinical efficacy of TMZ. ${ }^{23,24}$ Studies have shown that elderly GBM patients have rates of $M G M T$ promoter methylation similar to that in younger patients, ranging from $40 \%$ to $60 \%{ }^{25}$ Reifenberger et $\mathrm{al}^{20}$ performed a large, prospective, observational study of 233 elderly GBM patients with the objective of determining outcomes based on MGMT methylation status. The results revealed that patients who had tumors that exhibited $M G M T$ methylation had significantly longer OS than patients who had tumors without $M G M T$ promoter methylation (median OS: 8.4 months vs 6.4 months, $P=0.031$ ). The authors concluded that $M G M T$ promoter methylation might be a useful biomarker to stratify elderly GBM patients. ${ }^{20}$ Similar results have been observed in younger populations. ${ }^{23,24}$ These findings suggest that $M G M T$ methylation is an important factor determining outcomes in GBM patients with good performance status. The ANOCEF trial enrolled 70 elderly GBM patients with poor performance status and found that patients with tumors with $M G M T$ methylation had significantly longer survival than those with unmethylated tumors (7.75 months vs 4.5 months). Multivariate analysis also suggested that $M G M T$ methylation status was an independent prognostic factor in the TMZ group. ${ }^{6}$ Thus, the use of TMZ in elderly GBM patients was safe and showed favorable clinical efficacy. MGMT promoter methylation may be a useful and predictive biomarker of TMZ efficacy to stratify elderly GBM patients for treatment.

In the present study, multivariate analysis revealed that the extent of resection and KPS score were independent prognostic factors, which is in accordance with previous studies. ${ }^{26-28}$ In a retrospective study with post hoc analysis of data from a prospective randomized Phase III trial, Stummer et $\mathrm{al}^{28}$ reported that patients who underwent complete resection had significantly better prognosis than those who underwent incomplete resection (16.9 months vs 11.8 months; $P<0.001$ ), and multivariate analysis also showed that postoperative residual tumor was a significant predictor of poor prognosis. ${ }^{28}$

In our study, the toxicity profile of TMZ in elderly GBM patients was acceptable, even in those with impaired KPS. The most frequent toxicity documented was myelosuppression, which is in line with a previously reported series in elderly patients. ${ }^{29,30}$ Overall, elderly patients with impaired KPS may be able to tolerate TMZ chemotherapy well.

There are several inherent limitations in this study. The current study is unavoidably limited by its retrospective nature and the fact that it was conducted in a single institution. The propensity score-matching analysis could mitigate bias in the baseline characteristics, but this analysis cannot replace a prospective randomized trial. Furthermore, unknown or unmeasured confounders could not be taken into account, and this might lead to some residual bias.

\section{Conclusion}

Treatment with TMZ was a more effective option than BSC, with tolerable toxicity in elderly patients with GBM and impaired KPS.

\section{Disclosure}

The authors report no conflicts of interest in this work.

\section{References}

1. Ostrom QT, Gittleman H, Liao P, et al. CBTRUS statistical report: primary brain and central nervous system tumors diagnosed in the United States in 2007-2011. Neurooncology. 2014;16:1-63.

2. Chakrabarti I, Cockburn M, Cozen W, Wang YP, Preston-Martin S. A population-based description of glioblastoma multiforme in Los Angeles County, 1974-1999. Cancer. 2005;104:2798-2806. 
3. Stupp R, Brada M, van den Bent MJ, Tonn JC, Pentheroudakis G; ESMO Guidelines Working Group. High-grade glioma: ESMO Clinical Practice Guidelines for diagnosis, treatment and follow-up. Ann Oncol. 2014;25:93-101.

4. Malmstrom A, Grønberg BH, Stupp R, et al. Glioblastoma (GBM) in elderly patients: a randomized phase III trial comparing survival in patients treated with 6-week radiotherapy (RT) versus hypofractionated RT over 2 weeks versus temozolomide single-agent chemotherapy (TMZ). J Clin Oncol. 2010;28(Suppl; abstr LBA2002):180s.

5. Stupp R, Mason WP, van den Bent MJ, et al; European Organisation for Research and Treatment of Cancer Brain Tumor and Radiotherapy Groups; National Cancer Institute of Canada Clinical Trials Group. Radiotherapy plus concomitant and adjuvant temozolomide for glioblastoma. N Engl J Med. 2005;352(10):987-996.

6. Gállego Pérez-Larraya J, Ducray F, Chinot O, et al. Temozolomide in elderly patients with newly diagnosed glioblastoma and poor performance status: an ANOCEF phase II trial. J Clin Oncol. 2011; 29(22):3050-3055.

7. Malmström A, Grønberg BH, Marosi C, et al. Temozolomide versus standard 6-week radiotherapy versus hypofractionated radiotherapy in patients older than 60 years with glioblastoma: the Nordic randomised, phase 3 trial. Lancet Oncol. 2012;13(9):916-926.

8. Charlson M, Szatrowski TP, Peterson J, Gold J. Validation of a combined comorbidity index. J Clin Epidemiol. 1994;47(11):1245-1251.

9. Esteller M, Hamilton SR, Burger PC, Baylin SB, Herman JG. Inactivation of the DNA repair gene O6-methylguanine-DNA methyltransferase by promoter hypermethylation is a common event in primary human neoplasia. Cancer Res. 1999;59(4):793-797.

10. Minniti G, Scaringi C, Lanzetta G, et al. Standard (60 Gy) or shortcourse (40 Gy) irradiation plus concomitant and adjuvant temozolomide for elderly patients with glioblastoma: a propensity-matched analysis. Int J Radiat Oncol Biol Phys. 2015;91(1):109-115.

11. Gray RJ. A class of k-sample tests for comparing the cumulative incidence of a competing risk. Ann Stat. 1988;16:1141-1154.

12. Counsell CE, Grant R. Incidence studies of primary and secondary intracranial tumors: a systematic review of their methodology and results. J Neurooncol. 1998;37(3):241-250.

13. Arvold ND, Reardon DA. Treatment options and outcomes for glioblastoma in the elderly patient. Clin Interv Aging. 2014;9:357-367.

14. Karran A, Blake P, Chan D, et al. Propensity score analysis of oesophageal cancer treatment with surgery or definitive chemoradiotherapy. Br J Surg. 2014;101(5):502-510.

15. Corry J, Smith JG, Wirth A, Quong G, Liew KH. Primary central nervous system lymphoma: age and performance status are more important than treatment modality. Int J Radiat Oncol Biol Phys. 1998;41: $615-620$

16. Glantz M, Chamberlain M, Liu Q, Litofsky NS, Recht LD. Temozolomide as an alternative to irradiation for elderly patients with newly diagnosed malignant gliomas. Cancer. 2003;97(9):2262-2266.
17. Babu R, Komisarow JM, Agarwal VJ, et al. Glioblastoma in the elderly: the effect of aggressive and modern therapies on survival. J Neurosurg. 2016;124(4):998-1007.

18. Wick W, Platten M, Meisner C, et al; NOA-08 Study Group of Neuro-oncologyWorking Group (NOA) of German Cancer Society. Temozolomide chemotherapy alone versus radiotherapy alone for malignant astrocytoma in the elderly: the NOA-08 randomised, phase 3 trial. Lancet Oncol. 2012;13(7):707-715.

19. Yin AA, Cai S, Dong Y, et al. Ameta-analysis of temozolomide versus radiotherapy in elderly glioblastoma patients. J Neurooncol. 2014;116(2):315-324

20. Reifenberger G, Hentschel B, Felsberg J, et al; German Glioma Network. Predictive impact of MGMT promoter methylation in glioblastoma of the elderly. Int J Cancer. 2012;131:1342-1350.

21. Reyes-Botero G, Honnorat J, Chinot OL, et al. Temozolomide plus bevacizumab in elderly patients with newly diagnosed glioblastoma and poor performance status: an Anocef Phase II trial. J Clin Oncol. 2013;31(15 Suppl):2020.

22. Hegi ME, Diserens AC, Godard S, et al. Clinical trial substantiates the predictive value of O-6-methylguanine-DNA methyltransferase promoter methylation in glioblastoma patients treated with temozolomide. Clin Cancer Res. 2004;10(6):1871-1874.

23. Esteller M, Garcia-Foncillas J, Andion E, et al. Inactivation of the DNA repair gene $M G M T$ and the clinical response of gliomas to alkylating agents. N Engl J Med. 2000;343(19):1350-1354.

24. Hegi ME, Diserens AC, Gorlia T, et al. MGMT gene silencing and benefit from temozolomide in glioblastoma. $N$ Engl J Med. 2005; 352(10):997-1003.

25. Jordan JT, Gerstner ER, Batchelor TT, Cahill DP, Plotkin SR. Glioblastoma care in the elderly. Cancer. 2016;122(2):189-197.

26. Kuhnt D, Becker A, Ganslandt O, Bauer M, Buchfelder M, Nimsky C. Correlation of the extent of tumor volume resection and patient survival in surgery of glioblastoma multiforme with high-field intraoperative MRI guidance. Neuro Oncol. 2011;13(12):1339-1348.

27. Lacroix M, Abi-Said D, Fourney DR, et al. A multivariable analysis of 416 patients with glioblastoma multiforme: prognosis, extent of resection, and survival. J Neurosurg. 2001;95:190-198.

28. Stummer W, Reulen HJ, Meinel T, et al. Extent of resection and survival in glioblastoma multiforme: identification of and adjustment for bias. Neurosurgery. 2008;62:564-576.

29. Gzell C, Wheeler H, Guo L, Kastelan M, Back M. Elderly patients aged 65-75 years with glioblastoma multiforme may benefit from long course radiation therapy with temozolomide. J Neurooncol. 2014; 119:187-196.

30. Hoffermann M, Bruckmann L, Kariem Mahdy A, Asslaber M, Payer F, von Campe G. Treatment results and outcome in elderly patients with glioblastoma multiforme - a retrospective single institution analysis. Clin Neurol Neurosurg. 2015;128:60-69.
OncoTargets and Therapy

\section{Publish your work in this journal}

OncoTargets and Therapy is an international, peer-reviewed, open access journal focusing on the pathological basis of all cancers, potential targets for therapy and treatment protocols employed to improve the management of cancer patients. The journal also focuses on the impact of management programs and new therapeutic agents and protocols on

\section{Dovepress}

patient perspectives such as quality of life, adherence and satisfaction. The manuscript management system is completely online and includes a very quick and fair peer-review system, which is all easy to use. Visit http://www.dovepress.com/testimonials.php to read real quotes from published authors. 